\title{
A SIMPLE STAINING METHOD FOR THE VISUALISATION OF METACERCARIAE IN SMALL FISH AND TADPOLES
}

\author{
Gábor Majoros
}

Central Veterinary Institute, Tábornok u. 2, H-1149 Budapest, Hungary

There are several kinds of larval trematodes that can get into very young fish fry placed into fish ponds and cause illness or death in their hosts. An effective way to investigate these metacercariae is to prepare the living parasites fresh from the bodies of hosts, but occasionally the need arrises to inspect dead and fixed fish for the presence of the larvae. In this case, the fixed metacercariae are not identified as easily or as accurately as the living ones. Nevertheless, it is useful to determine the intensity and the abundance of the metacercarial infection in this case, too. In order to obtain information about the quantity and the distribution of metacercariae in fish, the entire bodies must be searched for the larvae.

Histological methods have been traditionally used for the detection of digenean trematode larvae in small fish (e.g. Rees G. 1957: Parasitology 47: 126-137; Ratanarat-Brockelman C. 1968: Z. Parasitenkd. 43: 123-134; Hendrickson G.L. 1978: Proc. Helminthol. Soc. Wash. 45: 64-68). In order to get an approximation of the number and location of larvae within a fish, a series of histological sections must be prepared from the infected animal (Hendrickson G.L. 1979: Exp. Parasitol. 48: 245-248). Histological techniques are time-consuming and expensive and not all larvae can be found in histological sections, unless the whole fish is sectioned carefully and the sections are arranged in series. For this reason some parasitologists have tried to develop methods to demonstrate metacercariae in the entire fish (Rau M.E., Gordon D.M. 1977: Can. J. Zool. 55: 1200-1201).

In order to avoid the above-mentioned problems, a new method is presented here for observing of metacercariae in small fish. (For larger fish this procedure can be used only on small pieces of their dissected organs or slices of their body, not thicker than $5 \mathrm{~mm}$.) This staining method consists of the following steps:

1. Small (maximum $2-3 \mathrm{~cm}$ long) fish or tadpoles are fixed in $4 \%$ formaldehyde buffered with phosphate buffer $(\mathrm{pH} 7.0)$ for at least 24 hours. If the fixation time lasts slightly longer than that, the effectiveness of the procedure is not reduced. However, in less concentrated ( $2 \%$ ) formaldehyde, specimens can be stored up to several months without a substantial change in the subsequent staining quality.

2. Fixed animals are bathed in distilled water for 1-2 hours to remove the formaldehyde. Changing the water twice is satisfactory.

3. The animals are then transferred into Mayer's haemalum stock solution (Kiernan J.A. 1990: Histological and Histo- chemical Methods - Theory and Practice. Pergamon Press, 433 pp.) mixed with $90-95 \%$ distilled water.

The animals are left in the haemalum mixture until they assume an opaque purplish-black colour. Depending on the mass of the animals and the permeability of their integument, intense staining can occur in 4-5 hours, but occasionally it may take a whole day. Specimens should be markedly overstained so that the staining solution can penetrate their entire body, thus reaching the internal tissues. If the fish or tadpoles are left in the staining solution for one or two days, steps should be taken to avoid alkalisation of the solution and precipitation of the stain. The staining dish should be covered to prevent the absorption of carbon dioxide and excessive evaporation.

4. The animals are then transferred to containers with plenty of tap water and soaked for 12 hours. To speed up the drenching process, a few drops of saturated caustic soda $\left(\mathrm{Na}_{2} \mathrm{CO}_{3}\right)$ solution is added to the tap water. The preparations should be stained deep blue by the end of this period.

5. The specimens are immersed in a $10 \%$ lactic acid solution which re-dissolves the haematoxylin from the tissues (i.e., differentiates), causing the animals to fade and turn red. Differentiation with lactic acid lasts at most a few minutes and needs to be checked under a dissecting microscope until the encapsulated or free metacercariae become visible in the host tissue, i.e., extraction of the stain continues until the parasite larvae, which destain more slowly, become distinguishable from the host tissues. Differentiation is based on the principle that lactic acid finds its way into the host cells more easily than it penetrates the parasite through the syncytial tegument or the walls of the cyst of the metacercaria. In addition, as the cell nuclei are more densely located in the tissues of parasites than hosts, the parasites retain their colour longer than do host organs.

6. When the hosts are cleared and trematode larvae can be discerned, lactic acid treatment is very quickly discontinued by washing the animals with distilled water once and then placing them in tap water containing two or three drops of saturated $\mathrm{Na}_{2} \mathrm{CO}_{3}$ solution. The elution of haematoxylin is stopped and the stain becomes dark bluish-purple again with a reddish tint. This second "bluing" phase takes approximately 10-20 minutes, depending on the body mass of the host, and is followed by rinsing in tap-water again to remove all traces of soda. 


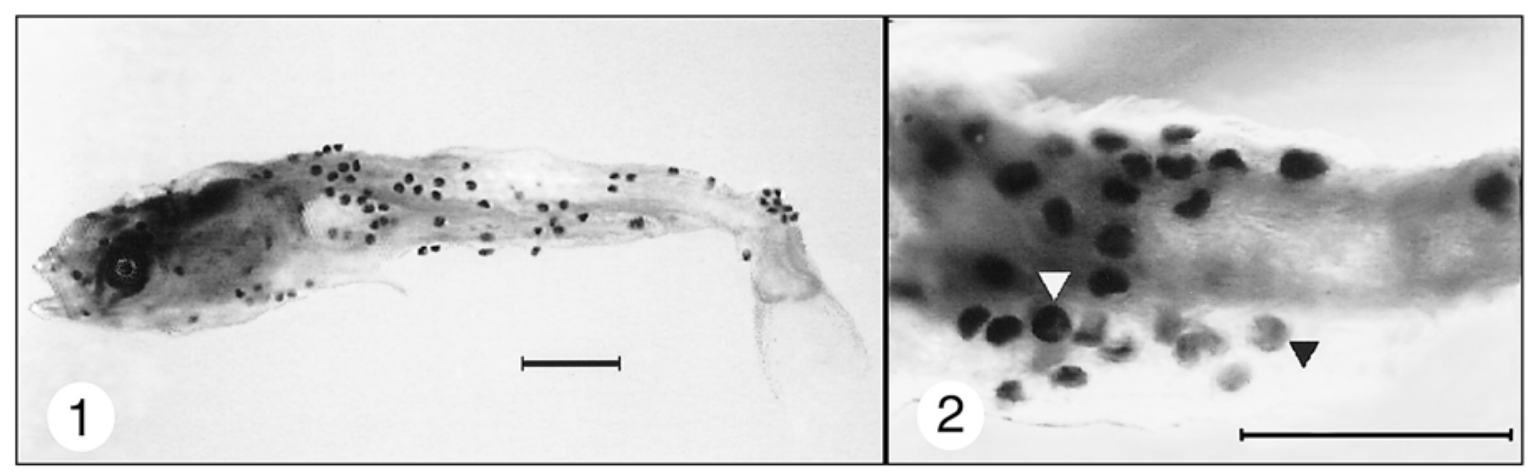

Fig. 1. Penetrated metacercariae (black spots) of an Opisthioglyphe species in a 7-day-old carp (Cyprinus carpio) fry. Haemalum staining, illuminated from above and from below. Fig. 2. Encapsulated $(\boldsymbol{\Delta})$ and freshly penetrated (unencapsulated) $(\Delta)$ metacercariae of an Opisthioglyphe species in a 7-day-old carp (C. carpio) fry. Staining and illumination as for Fig. 1. Scale bars $=1 \mathrm{~mm}$.

7. To increase the contrast between the larvae and the host tissues, the fish or tadpoles are cleared using a lower degree of polycondensation type polyethylene glycol (polyoxaethene, macrogol) which is liquid at room temperature even without dissolving in water. Therefore samples are placed in a $25 \%$ solution of Macrogolum-400 (Ph. Hg. VI.; Merck) in distilled water for one week. Subsequently, the samples are kept first in a $30 \%$ and then in a $50 \%$ solution of the same substance for one week each. To achieve more permanent preservation, fish processed in this way can be carried through a graded series of Macrogolum-400 solutions of increasing concentration, at a similar rate (e.g., 70-90-100\%), and they can be stored in $100 \%$ polyethylene glycol at room temperature. Alternatively, processed animals can be stored in 50\% polyethylene glycol for a relatively long time if bacterial deterioration of the solution is prevented by the addition of some mild, compatible preservative (e.g., 1-5\% methanol, ethanol, isopropanol or a few crystals of sodium benzoate) or by storage in a refrigerator. However, this is not necessary for rendering the fish transparent, as maximum transparency is reached in the $50 \%$ solution.

During the procedure, the parasite larvae are seen as light or dark purple spots against the almost colourless background of the surrounding tissues (Fig. 1). Although tissues become slightly more rigid compared to their natural state, they remain suitable for dissection. If the body of a host is so large that the whole animal does not become completely translucent, its organs become transparent and observable only after autopsy. Should we need to make an autopsy after the clearing process, to decrease the rigidity of the tissues, polyethylene glycol can be washed from them with water. The preparations also remain suitable for embedding in paraffin, as they can be easily dehydrated using intermediary substances (e.g., graded series of ethanol). This also means that they can also be saturated with clove oil or methyl salicylate to achieve a higher degree of clarity. (However these materials cause the bodies to become rigid irreversibly.)
After fixation, it is advisable to decalcify fish containing ossified tissues with $1 \%$ Na-EDTA. This does not disturb the later phases of the procedure and even prevents the formation of $\mathrm{CO}_{2}$ bubbles that might appear during subsequent lactic acid treatment. Decalcification is also useful in making the intestinal contents transparent, primarily in the case of tadpoles that have a long gut. The pigments of the host organism can be rendered colourless by treatment with $10 \%$ hydrogen peroxide (Kiszely G., Barka T. 1958: Practical Micro-techniques and Histochemistry. Medicina, Budapest, $346 \mathrm{pp}$. [in Hungarian]) or 2\% Javel's lye ( $\mathrm{KOCl})$ (personal observation); however, the excessive use of the latter considerably degrades the cysts of metacercariae and, thus, may inhibit selective staining.

If soaking in polyethylene glycol is performed more rapidly than described above, this may cause the preparation to shrink. Although this may be objectionable from an aesthetic point of view, it does not markedly disturb the detection of trematode larvae. Therefore, in routine mass examinations, the soaking time can be shortened to one or two days or, alternatively, more concentrated solutions can be used from the beginning. If the preparations are placed into a $50^{\circ} \mathrm{C}$ incubator or a microwave oven producing a similar temperature, they will more rapidly become saturated with the clearing solution; however, this latter method requires constant supervision to prevent raising the temperature too high. Tween- 80 or similar detergents mixed with polyethylene glycol do not appreciably accelerate the process of saturation. Saturation can be considered to have occurred when the preparation, originally floating on the surface of the solution, sinks to the bottom.

Once they are rendered transparent, the preparations can easily be photographed in a dark field under oblique illumination. Figs. 1 and 2 show examples of Opisthioglyphe metacercariae that have penetrated a carp fry.

The procedure presented above proved to be successful in the detection of Diplostomum, echinostome metacercariae and 
metacercariae of different xiphidiocercariae in naturally infected 110 carp (Cyprinus carpio L.) fry (2-3 cm in body length), 30 juvenile guppies (Poecilia reticulata Peters) and 8 tadpoles (Rana $\times$ esculenta). Even the cysts of the ciliate Ichthyophthirius multifiliis Fouquet, 1876 were clearly detectable in dead fish fry in a similar way following this procedure. An advantage of this method is that it enables an inexpensive routine examination of a large series of fish fry needed to provide sufficient data for statistical evaluation. Thus, the ratio of mortality due to penetrating cercariae can be quantified and differentiated from other factors causing fish fry mortality, and the seasonality of larval trematode invasions can be readily studied in fish farming situations.

Accepted 3 August 2000 